\title{
A feeding frenzy for embryos and stem cells: meeting the media frontally!
}

\author{
David F. Albertini
}

Published online: 26 August 2011

(C) Springer Science+Business Media, LLC 2011

With all the media coverage over the obesity epidemic of late, it comes as no surprise that the dietary habits of embryos and stem cells are drawing mounting attention in the fields of reproductive, as well as regenerative medicines. A not-so-obvious parallel between the diets of human embryos and stem cells was perhaps unavoidable, when considering culture conditions that, in the end, provide both disciplines with their outcomes-embryos capable of establishing and maintaining a pregnancy or pluripotent stem cells that could be transformed into tissue replacements. The feeding frenzy at hand derives from recent studies that, on the one hand, revisit the question of how culture media influence the birth of embryonic stem cells within the inner cell mass and on the other, seek to establish chemically defined conditions for deriving and maintaining induced pluripotent stem cells(iPSC).

Obtaining chemically defined media for use in human embryo or stem cell cultures has been a lofty goal for the biomedical research community for many years. In the field of human ARTs, reducing the complexity of human serum has led to the emergence of a host of protein supplements designed primarily to replace animal products of any kind and that, secondarily, would satisfy the palatal desires of an embryo hell-bent on parasitizing a uterus. Deciding whether media optimization should entail a one-course or multiplecourse menu of options for buffering, protein, anti-oxidants and so on, has been the stuff of much debate and continues to occupy the attention of ART practitioners. Fueling the

Capsule Advances in stem cell biology offer insights into present and future perspectives for human embryo culture.

D. F. Albertini $(\bowtie)$

Kansas University Medical Center,

Kansas City, KS 66160, USA

e-mail: dalbertini@kumc.edu preimplantation embryo for the most commonly deployed 3- or 5-day culture periods prior to embryo transfer must entail a menu suitable for sustaining two events. First, establishing a trophectoderm with an appetite (metabolism) of voracious proportions. And second, sequestering a subset of cells within the inner cell mass, from which embryonic stem cells will arise and which, by all (metabolic) accounts, recognizes the importance of small proportions. It is this divergence in dietary habits, founded in the distinct metabolic demands of trophectoderm and inner cell mass cells, which brings us to our lead article this month.

With efforts having focused over the past 10 years on achieving chemically defined conditions that approximate the various tubal and uterine environments encountered by the early embryo, a different perspective on an old problem is welcoming. The paper by Gelber and colleagues challenges conventional wisdom by investigating the utility of embryonic stem cell media in the production of preimplantation embryos. They draw upon the gold standard for testing their hypothesis - the mouse embryo - and, in the course of their work, methodically trace not only developmental fates of embryos prior to implantation, but closely examine the expression of trophectoderm and inner-cell mass markers in the context of cell allocation (see cover). While the bar is set high because these studies use in vivo- (not in vitro-) produced embryos, the results are sure to raise eyebrows when it comes to reactivating the debate over simple versus complex media for ARTs.

Their studies are a two-way comparison between KSOM and embryonic stem cell medium. When in vivo produced 2-cell embryos are cultured under either condition, the findings suggest that blastocyst quality is indeed superior in KSOM as would be expected, given the current status quo. On the other hand, when 8-cell embryos are used as starting material, there is a striking improvement in blastocyst 
quality and lineage biomarker expression in the group cultured in embryonic stem cell medium (ESCM) relative to KSOM. For example, increased expression levels for both OCT 4 protein and mRNA are observed after ESCM, when compared to KSOM. These findings raise several questions: Does ESCM, as it was originally designed to do, tip the balance towards more robust proliferation of inner cells, whilst repressing the expression of trophectoderm markers such as CDX2? Is the displacement of cells away from the trophectodermal lineage problematic with respect to initiating and sustaining implantation? And finally, would the effects of cell allocation observed presently in mice be recapitulated in human embryos and if they were, how would this impact the derivation and utility of new lines of human embryonic stem cells?

These provocative findings arrive at an important juncture in the field of stem cell biology. With the advent of iPS technology pioneered and recently advanced by Yamanaka and colleagues (see Okita et. al., Nature Methods, 2011;DOI:10.1038/NMETH.1591), comes the opportunity to produce cells of therapeutic value in an efficient and safe manner. Thus, the discovery of chemically defined conditions for producing and propagating iPSC by another pioneering laboratory, that of Jamie Thomson's, bears witness to the need to overcome technological obstacles in the stem cell field, obstacles that may have application in the long run for human ARTs. To wit, the studies of Chen et. al. (Nature Methods, 2011; DOI:10.1038/NMETH.1593) introduce E8 medium and eliminate one of the major sources of inconsistencies in stem cell propagation that had been attributed to serum albumin or substitutes thereof. This new medium takes advantage of vitronectin-coated substrates and protein supplements in achieving chemical definition and improved derivation efficiencies for iPSCs and ESCs. Notably, albumin is eliminated and transferrin, FGF2, and TGFbeta are sufficient protein additives shown to be effective for derivation and maintenance of stem cells on a background of small chemical inhibitors that tweak the system in a favorable direction.

In view of the strides made in this field, as evidenced above, perhaps it is time for those of us in the arena of human ARTs to take notice of these advances and consider the likelihood of gustatorial commonalities between the human embryo and stem cells, whether they be of iPSC or embryonic origins. 